\title{
TITLE:
}

\section{On the coevolution of the product quality and the consumer preferences}

$\operatorname{AUTHOR}(S)$ :

Ida, Takanori

\section{CITATION:}

Ida, Takanori. On the coevolution of the product quality and the consumer preferences. 京都大学大学院経済学研究科Working Paper 2003, 66

\section{ISSUE DATE:}

2003-01

URL:

http://hdl.handle.net/2433/37945

RIGHT: 


\section{On the Coevolution of the Product Quality} and the Consumer Preferences

\section{Takanori Ida}

Graduate School of Economics, Kyoto University, Japan

January, 2003

Graduate School of Economics

Faculty of Economics

Kyoto University

Kyoto, 606-8501 JAPAN 
On the Coevolution of the Product Quality and the Consumer Preferences

\author{
Takanori Ida \\ Graduate School of Economics, Kyoto University, Japan
}

January, 2003 


\title{
On the Coevolution of the Product Quality
}

\author{
and the Consumer Preferences
}

\section{Takanori Ida}

Faculty of Economics, Kyoto University, Japan

\begin{abstract}
This paper will analyze the self-reinforcing coevolutionary process of innovation, based on the framework of evolutionary ecology and population genetics. In particular, central to this analysis is Fisher's runaway process, which demonstrates the coevolution of the quality of products and the preferences of consumers in our context. The four main points this paper seeks to make are as follows. First, it can be concluded from a matching model of supply and demand that when a consumer who has a preference for high quality discovers a product of high quality in the market, he/she will certainly purchase that product. Second, taking account of both the high survival rate of a firm that supplies a product of high quality and the cost to the firm of improving the quality, evolutionarily stable Fisher's process can be explained. Third, however, considering the misfortune of a consumer who has a too-high preference, Fisher's process will disappear. Fourth, with the existence of power-users or the effect of the negative bias of innovation, Fisher's process can be recovered.
\end{abstract}

Keywords: innovation, evolution, runaway process, startup problem

JEL Classification: L13, L51, L86

\author{
Address: \\ Takanori Ida \\ Faculty of Economics, Kyoto University, \\ Yoshida, Sakyo, Kyoto 606-8501, Japan \\ Tel\&Fax: +81- 75-753-3477 \\ E-mail: ida@econ.kyoto-u.ac.jp
}




\section{Introduction}

It is evolutionary economics that studies a mechanism that introduces novelties, innovations, and inventions to an economic system and also discusses the cumulative causation processes, the lock-in phenomena, and the path dependence ${ }^{1}$. An important concept in evolutionary economics is 'population thinking' (see Mayr 1959, Metcalfe 1998). This claims that a variety of characteristics within a population are the prerequisite for evolutionary change; thus the statistical moments of the population distributions of characteristics, such as mean, variance, and covariance, and their rates of change over time provide the measures of the rate and direction of evolution. This paper will deal with the evolutionary development of innovation, based on the population thinking.

The process of the diffusion of innovation is often regarded as a startup problem $^{2}$. The startup problem is similar to a chicken-and-egg problem between

\footnotetext{
${ }^{1}$ Following classical evolutionary economists such as Marshall, Veblen, Schumpeter, Alchian, a number of modern evolutionary economists have recently emerged. Typical examples are Nelson and Winter (1984), Dosi et al. (1988), Hodgson (1994), Witt (1993), Anderson (1994), Freeman (1982), Antonelli and De Liso (1997), Dosi (2000), Andersen (2001). According to Grupp (1998), there are three assumptions in modern evolutionary economics: (1) limitedly rational players negotiate at the micro-level; (2) transactions are not equilibrium oriented and can therefore occur away from equilibrium state; and (3) markets and other facilities define the selection mechanisms between heterogeneous institutions and technical facilities.

${ }^{2}$ It is in fact a difficult question to precisely define what innovation is. According to Sundbo (1998), innovation can be classified into the following types: (1) a new product or a new service, (2) a new
} 
supply and demand: consumers attach no value to products unless the sufficient supply of the products of good quality in the future is guaranteed, while suppliers of the products do not want to provide them unless the sufficient demand in the future is not expected. A question now arises: which should precede the development of innovation, supply or demand? The answer is that the interaction of both is necessary. Without the positive interaction between the supply-side and the demand-side, new technologies or new services will not become more wide spread.

There are many failures in the development of innovation. On the one hand, we know new services whose technologies were almost completed but which failed to take off because they could not stimulate the initial demand sufficiently. Examples of this type are Picturephone by AT\&T, Minitels by France Telecom, N-ISDN by NTT and so on (cf. Rohlfs 2001). On the other hand, there are new technologies whose potential demands were large enough but whose technical difficulties could not be solved, so that they have not yet been able to break through. Examples of them are electro-mobile, linear-motor-car, and alternative energy of petroleum.

However, the few but impressive successes of the interactions of supply and demand should not be forgotten. Consumers always tend to demand new technologies/services of higher quality. Responding to the strong demands of production process, (3) a new organizational or management structure, and (4) a new type of marketing or overall behavior in the market. 
consumers, suppliers try to provide innovative technologies/services. Since consumers are willing to purchase such high quality products within their budget constraints, suppliers who succeed in developing them will very likely survive the market competition and furthermore try to develop products of even higher quality. On the other hand, consumers who have preferences for higher quality are satisfied with the purchase of such products and demand the further improvements of technologies/services. As a result, a series of innovations will be constantly encouraged. Examples of these are the performance of computer's MPU and the broad-bandwidth of data communication. 'Mecalfe's law' and 'Moore's law' are interesting hypotheses that describe the successful developments of technologies/services: the former claims that the value of a user grows proportionally with the square of the number of users, while the latter suggests that the computing performance-to-price ratio doubles every 18 months. Of course, although they are not scientific laws in a strict sense, they seem to aptly demonstrate the successful coevolution of supply and demand in the current information-communication services.

It will be interesting to analyze the self-reinforcing coevolutionary process of innovation, based on the framework of population genetics (cf. Falconer 1981, Bulmer 1985). 'Fisher's law' in population genetics establishes the law of motion of average fitness, and thus of the relative frequencies of the related characteristics, as a function of the higher moments of the distribution of the latter. 'Fisher's runaway process' is very interesting and suggestive (cf. Fisher 1915). Male mating behavior often involves the 
display of extravagant ornamentation accompanied by loud and complex songs and the release of volatile secretions. To understand the evolution of these exaggerated secondary sexual characteristics, Fisher suggested that the advantage of preference lies in the choice of mates who will father attractive sons. If females on average prefer a particular male trait, then males with that trait have a mating advantage and, if the trait is heritable, females with the preference have male offspring similarly adorned who also enjoy a mating advantage. See Pomiankowski et al. (1991, p.1422) for further discussion. Metcalfe (1998) is a pioneer of those who apply Fisher's law to economics. Metcalfe's point can be summarized as follows. Different forms of organization can engender different levels of profitability, and these latter imply the different rate and direction of technological change. Assuming there is average technology in terms of costs or quality-adjusted price, the relative economic weight of a product, and thus of a technology, changes according to its distance from the average performance within the technology set.

The main points this paper seeks to make are as follows. First, it can be concluded from a matching model of supply and demand that when a consumer who has a preference for high quality discovers a product of high quality in the market, he/she will certainly purchase that product. Second, taking account of both the high survival rate of a firm that supplies a product of high quality and the cost to the firm of improving the quality, evolutionarily stable Fisher's runaway process can be explained. Third, however, considering the misfortune of a consumer who has a too-high 
preference for high quality, Fisher's process will disappear. Fourth, with the existence of power-users or the effect of the negative bias of innovation, Fisher's process can be recovered. In this way, this paper will investigate Fisher's runaway processes under various conditions and reach some interesting results. The paper consists of the following five sections. Section 2 describes a matting model. Section 3 sets up a basic model for describing the interaction between supply and demand. Section 4 analyzes various additional conditions, developing the basic model, whilst Section 5 draws a conclusion.

\section{Matting model}

In this section, a matting model between supply and demand will be established, and the relationship between the preference for quality and the purchase decision of consumers will be investigated. A consumer goes to the market, searches for various products, purchases a product if he/she is pleased with it or does not purchase it if he/she is not. One period ends when the consumer exits from the market because of the constraints of time and budget. To make the analysis simple, we assume that one firm supplies only one product. The timing of the model is depicted in Fig. 1.

$<$ Fig. 1 $>$ 
Basic definitions are given as follows:

$n$ kinds of products: $i=1, \ldots, n$

the quality of product $i: x_{i} ; 0 \leq x_{i}$

the probability that a consumer discovers product $i$ in the market in one period: $p_{i}$

the probability that a consumer purchases product $i$ when he/she discovers it: $q_{i}$ the strength of the preference of a consumer for the quality of products: $y ; 0 \leq y$ the utility which a consumer with preference $y$ gets from purchasing product $i$ :

$$
U_{i}=U\left(x_{i} \mid y\right) ; \quad \partial U_{t} / \partial x_{i}>0
$$

the cost which a consumer with preference $y$ pays for purchasing product $i$ :

$$
C_{i}=C\left(x_{i} \mid y\right) ; \quad \partial C_{i} / \partial x_{i}>0
$$

the unit utility of product $i$, defined as the utility divided by the cost:

$$
W_{i}=W\left(x_{i} \mid y\right)=U_{i} / C_{i}
$$

We suppose that a consumer determines the bundle of probabilities, $\left(q_{1}, \ldots, q_{n}\right)$, representing whether he/she should purchase products or not when he/she discovers them in the market. Letting the operator of expectation be $E$, the expected unit-utility that the consumer gets from consuming product $i$ in a particular period of time is expressed as follows:

$$
E W\left(q_{1}, \ldots, q_{n}\right)=\frac{\sum_{i} p_{i} q_{i} U_{i}}{\sum_{i} p_{i} q_{i} C_{i}}
$$

The numerator of Eq. (1) means the expected utility, and the denominator, the expected cost. To calculate the bundle of probabilities, $\left(q_{i}{ }^{*}, \ldots, q_{n}{ }^{*}\right)$, which maximizes the 
expected unit-utility, the partial derivative of the expected unit-utility with respect to $q_{k}$ is given as follows:

$$
\frac{\partial E W}{\partial q_{k}}=\frac{p_{k} C_{k}}{\sum_{i} p_{i} q_{i} C_{i}}\left(\frac{U_{k}}{C_{k}}-E W\right) .
$$

The term $q_{k}$ is a probability lying in $[0,1]$; thus the consumer will certainly purchase product $k$, namely $q_{k}=1$, if its unit utility, $U_{k} / C_{k}$, is larger than the expected unit-utility, $E W$. The converse holds for $q_{k}=0$. Supposing $U_{1} / C_{1}>U_{2} / C_{2}>\ldots>U_{n} / C_{n}$ for the sake of simplicity, we obtain $q_{k}{ }^{*}=1$ and $q_{k+1}{ }^{*}=0$ for $k$ such as $U_{k} / C_{k}>E W\left(q_{1}{ }^{*}, \ldots, q_{n}{ }^{*}\right)>U_{k+1} / C_{k+1}$.

We assume here that the unit utility of product $i$ increases with its quality, namely $\partial W_{i} / \partial x_{i}>0$. Given $W_{i}=U_{i} / C_{i}$, this assumption can be replaced by the following inequality:

$$
\frac{\partial U_{i} / \partial x_{i}}{U_{i} / x_{i}} / \frac{\partial C_{i} / \partial x_{i}}{C_{i} / x_{i}}>1
$$

The left-hand side of Eq. (3) represents the elasticity of utility to cost, and it follows that unit utility is an increasing function with respect to quality if the value of the elasticity is larger than 1. Since parameter $y$ denotes the strength of the preferences of consumers for quality, we may assume that the elasticity is an increasing function with respect to $y$, namely $\partial^{2} W_{i} / \partial x_{i} \partial y>0$. Consequently, $\partial W_{i} / \partial x_{i}>0$ holds for a sufficiently high level of preference $y^{*}$. For example, letting the utility function be $U_{i}(x \mid y)=x_{i}^{y}$ and the cost function be $C_{i}(x \mid y)=c x_{i}$, the condition that the elasticity of the unit utility is larger than 1 is $y^{*}>1$. From these remarks, the following result is obtained: 
Proposition 1. When a consumer who has a preference for high quality discovers a product of higher quality than some specific level, he/she will certainly purchase that product. On the other hand, when he/she discovers a product of lower quality than some specific level, he/she will never purchase that product.

Based on the proposition above, we will consider the dynamic interaction between supply and demand. Since a consumer has a preference for high quality, he/she purchases a product not of low quality but of high quality. Thus a firm that supplies a product of high quality can survive the market competition and tries to develop a product of even higher quality. By contrast, a firm that supplies a product of poor quality cannot survive and must be selected through the market mechanism. Therefore, the quality of products tends to improve and, at the same time, the required level by consumers tends to rise. A process in which both quality and preference evolve is called here 'Fisher's runaway process' after its advocate; Fig. 2 depicts Fisher's process. In the following section, we will establish the model for analyzing the dynamic interaction between supply and demand.

$<$ Fig. 2>

\section{Dynamic model I}


In this section, we will propose a model for describing the interaction between supply and demand. To begin with, a fundamental equation which describes the dynamics of the average values of the quality of products and the preferences of consumers. Next, the dynamics of the survival rate of a firm will be examined.

\subsection{Fundamental equation}

Letting both the quality of product, $x$, and the preferences of consumers, $y$, be random variables, we will study a fundamental equation describing how their average values, $E_{x}$ and $E_{y}$, change over time. Added to the definitions given in the previous section, we define further variables as follows: let the variances of $x$ and $y$ be $V_{x}$ and $V_{y}$ and the covariance between $x$ and $y$ be $R$; let the survival rate of a firm that supplies a product of quality $x$ be $S_{x}$, and the level of satisfaction, in other words of the continuing rate of the consumption activity, of a consumer who has a preference $y$ be $D_{y^{*}}$ In this case, we can formalize the evolution of the two mean values, $E_{x}$ and $E_{y}$, by using the variances, $V_{x}$ and $V_{y}$, and the covariance, $R$, in the following equations:

$$
\begin{aligned}
& d E_{x} / d t=V_{x}\left(\partial \log S_{x} / \partial x\right)+R\left(\partial \log D_{y} / \partial y\right) \\
& d E_{y} / d t=R\left(\partial \log S_{x} / \partial x\right)+V_{y}\left(\partial \log D_{y} / \partial y\right) \\
& \text { evaluated at } x=E_{x}, y=E_{y} .
\end{aligned}
$$

Eqs. (4) are the same type as the fundamental equation concerning the additive genetic values in population genetics (cf. Lande 1981, Iwasa et al. 1991). See Appendix I for further detail. 


\subsection{Dynamic model with the survival rate of a firm}

At this point, we will analyze a dynamic model with the survival rate of a firm that supplies the product of quality $x$. It follows from Proposition 1 that the higher the quality of products, $x$, and the preferences of consumers, $y$, are, the higher the survival rate of the firm, $S_{x}$, is. Specifying the survival power of the firm by the product of $x$ and $y$, we assume that the survival rate, $S_{x}$, increases exponentially with the survival power, $x y$; on the other hand, we assume at this point that the continuing rate of the consumption activity is constant. The terms used below, $a, b, c, d, k$ and $l$, are parameters. To sum up, we obtain the following equations ${ }^{3}$ :

$$
\begin{aligned}
& S_{x}=k e^{a x y} \\
& D_{y}=l .
\end{aligned}
$$

Substituting Eqs. (5) into Eqs. (4), we obtain the following equations:

$$
\begin{aligned}
& d E_{x} / d t=a V_{x} E_{y} \\
& d E_{y} / d t=a R E_{y}
\end{aligned}
$$

The equilibrium of Eqs. (6) is the X-axis, $E_{y}=0$. The line of equilibrium is unstable, and thus $E_{x}$ and $E_{y}$ diverge endlessly in the first quadrant if they are out of equilibrium, as depicted in Fig. 3; see Appendix II for further analysis of the equilibrium. It is true that we can consider Fisher's runaway process as a process that diverges from the equilibrium, but it is unlikely that the preferences of consumers and the quality of products actually coevolve indefinitely. This strange result is based on the assumption

\footnotetext{
${ }^{3}$ Additionally, we need to assume $0 \leq S_{x} \leq 1$ and $0 \leq D_{y} \leq 1$, but at this point we do not take account of these conditions to make the analysis simple.
} 
that the survival rate, $S_{x}$, increases exponentially with the quality of product, $x$.

$<$ Fig. 3>

Then, we go on to suppose that firms have to pay the cost to improve the quality of products, $x$. Firms have to invest a lot of money in the R\&D activities, and the payment often bears down on the corporate finances and sometimes makes firms go bankrupt. Letting the negative effect of the payment on the survival rate of a firm be the square of the quality of product, $-x^{2}$, the following equations are obtained:

$$
\begin{aligned}
& S_{x}=k e^{a x y} e^{-b x^{2}} \\
& D_{y}=l
\end{aligned}
$$

Substituting Eqs. (7) into Eqs. (4), the following equations are obtained:

$$
\begin{aligned}
& d E_{x} / d t=V_{x}\left(a E_{y}-2 b E_{x}\right) \\
& d E_{y} / d t=R\left(a E_{y}-2 b E_{x}\right) .
\end{aligned}
$$

The equilibrium of Eqs. (8) is line $E_{y}=(2 b / a) E_{x}$. The stability of the equilibrium depends on parameter conditions: it is stable if $a / 2 b<V_{x} / R$, while unstable if $a / 2 b>V_{x} / R$; Fig. 4 illustrates the case of the former. Parameter $b$ means the degree of the effect of the cost to improve the quality of products. Thus there is an evolutionarily stable trajectory in which both $x$ and $y$ at first coevolve and then converge to the equilibrium, in the case where the payment parameter, $b$, is so large that the stability condition, $a / 2 b<V_{x} / R$, holds ${ }^{4}$. It is possible to interpret this case as an example of Fisher's

\footnotetext{
${ }^{4}$ We can suppose the cost for improving quality as other higher-order functions instead of quadratic function, $-x^{2}$. For example, assuming quartic function, $-x^{4}$, the equilibrium will become $E_{y}=(4 b / a) E_{x}^{3}$; it
} 
runaway process that is stably converging. See Appendix II for the further analysis of the equilibrium. The results can be summarized as follows:

$<$ Fig. 4>

Proposition 2. We have examined two effects on the survival rate of a firm that supplies the product of quality $x$ : the first tells us that the survival rate of the firm increases with the interaction of quality $x$ and preference $y$; the second informs us that it decreases with the payment for improving quality $x$. Either effect by itself cannot explain Fisher's runaway process in which both $x$ and $y$ at first coevolve and then converge to the equilibrium. On the other hand, both effects combine to be able to display that evolutionarily stable process.

The point here is that not only the interaction between quality $x$ and preference $y$ but also the cost for improving quality $x$ must be taken into consideration so that Fisher's process is regarded as a stably converging process towards the equilibrium.

\section{Dynamic model II}

is unstable if $x$ and $y$ are small while stable if $x$ and $y$ are large. 
In this section, we will expand the dynamic model, including the level of satisfaction of consumers, which was not considered in the previous section.

\subsection{Dynamic model with the level of satisfaction of consumers}

It is problematic to assume the level of satisfaction of consumers to be constant; for example, it may be that a consumer who has a too-high preference for quality cannot be as satisfied as other consumers who have lower preferences for quality because the consumer with the too-high preference hardly ever discovers a product corresponding to the preference in the market. . Letting the negative effect of the toohigh preference on the level of satisfaction, or the continuing rate of the consumption activity, of consumers be the square of the preference, $-y^{2}$, we obtain the following equations:

$$
\begin{aligned}
& S_{x}=k e^{a x y} e^{-b x^{2}} \\
& D_{y}=l e^{-c y^{2}}
\end{aligned}
$$

Substituting Eqs. (9) into Eqs. (4), we obtain the following equations:

$$
\begin{aligned}
& d E_{x} / d t=V_{x}\left(a E_{y}-2 b E_{x}\right)-2 c R E_{y} \\
& d E_{y} / d t=R\left(a E_{y}-2 b E_{x}\right)-2 c V_{y} E_{y} .
\end{aligned}
$$

The equilibrium of Eqs. (10) is the origin. This is because we obtain $\left(V_{x} V_{y}-R^{2}\right) E_{y}=O$ by setting $d E_{x} / d t=0$ and $d E_{y} / d t=0$; because of a rule between variance and covariance, $V_{x} V_{y^{-}}$ $R^{2}>0$ always holds; thus we see $E_{y}=0$; similarly we see $E_{x}=0$. Furthermore, turning to the analysis of the stability of the equilibrium, it follows that points out of the 
equilibrium necessarily converge to the equilibrium along line $a E_{y}-2 b E_{x}=0$, as depicted in Fig. 5; see Appendix II for further analysis of the equilibrium. It may be that we can interpret Fisher's runaway process as a temporally dis-equilibrium phenomenon until converging to the equilibrium, but such a process does not last for a long period ${ }^{5}$. An example is shown in Fig. 6, where both the average quality of products, $E_{x}$, and the average preference of consumers, $E_{y}$, increase only in the beginning short period, then start to decrease, and lastly converge to zero. From these remarks, the following result is obtained:

$<$ Figs. 5 and 6>

Proposition 3. When we consider the possibility that a consumer with a too-high preference is less satisfied, Fisher's runaway process that is evolutionarily stable disappears; at best, Fisher's process is regarded only as a temporally disequilibrium phenomenon.

\subsection{Conditions on which Fisher's process is recovered}

We have seen that evolutionarily stable Fisher's process disappears if we consider the misfortune of a consumer with a too-high preference. However, we

\footnotetext{
${ }^{5}$ This result is quite robust; insofar as we assume $D_{y}=l e^{-a y^{2}}$, the solution of this system is always $E_{y}=0$, regardless of the functional form of $S_{x}$
} 
actually know a few impressive successes among new technologies/services, which can be interpreted as examples of Fisher's process that is evolutionarily stable. Let us investigate conditions under which we can see Fisher's process at work again.

The first step is to suppose that there are consumers who can be contented with their having higher preferences than others, even if they cannot discover appropriate products corresponding to their preferences. This type of consumer can be regarded as a 'power-user,' who gets utility from such peculiar preferences. Taking account of the effect that the difference between an individual preference and the average preference of a population, $y-E_{y}$, increases the level of satisfaction of the consumers, $D_{y}$, we obtain the following equations:

$$
\begin{aligned}
& S_{x}=k e^{a x y} e^{-b x^{2}} \\
& D_{y}=l e^{-c y^{2}} e^{d(y-E y)} .
\end{aligned}
$$

Substituting Eqs. (11) into Eqs. (4), we obtain the following equations.

$$
\begin{aligned}
& d E_{x} / d t=V_{x}\left(a E_{y}-2 b E_{x}\right)+R\left(d-2 c E_{y}\right) \\
& d E_{y} / d t=R\left(a E_{y}-2 b E_{x}\right)+V_{y}\left(d-2 c E_{y}\right) .
\end{aligned}
$$

The equilibrium of Eqs. (12) is a non-zero point in the first quadrant, $\left(E_{x}=a d / 4 b c\right.$, $\left.E_{y}=d / 2 c\right)$. Furthermore, the equilibrium is stable; there is a trajectory in which both the average quality of products, $E_{x}$, and the average preference of consumers, $E_{y}$, at first increase, then slow down, and lastly converge to the equilibrium, as depicted in Fig. 7. An example of the coevolution of $E_{x}$ and $E_{y}$ over time is also shown in Fig. 8. 
$<$ Figs. 7 and $8>$

The second step is to suppose that innovation has an adverse effect (negative bias) on the survival rate of a firm that is engaged in innovative R\&D activities (cf. Pomiankowski et al. 1991 about the negative bias). Frequently, new technologies/services that firms have endeavored to develop cause consumers to lose interest in these products contrary to firms' intention and, in this way, have negative effects on the survival rate of firms. Taking account of this negative bias of innovation, $-u$, we obtain the following equations:

$$
\begin{aligned}
& d E_{x} / d t=V_{x}\left(a E_{y}-2 b E_{x}\right)-2 c R E_{y}-u \\
& d E_{y} / d t=R\left(a E_{y}-2 b E_{x}\right)-2 c V_{y} E_{y} .
\end{aligned}
$$

The equilibrium of Eqs. (13) is a point but the origin, $\quad\left[E_{x}=u\left(a R-2 c V_{y}\right) / 4 b c\left(V_{x} V_{y}-R^{2}\right)\right.$, $\left.E_{y}=(u / 2 c) R /\left(V_{x} V_{y}-R^{2}\right)\right]$; we see that $E_{y}>0$ always holds and $E_{x}>0$ holds in the case of $a / 2 c>V_{y} / R$. Furthermore, the equilibrium is stable; thus there is a trajectory in which both the average quality of products, $E_{x}$, and the average preference of consumers, $E_{y}$, at first increase, then slow down, and lastly converge to the equilibrium, given $a / 2 c>V_{y} / R$; we can depict them parallel to Figs. 7 and 8. From these remarks, the following result is obtained:

Proposition 4. If there are power-users who can directly get utilities from their higher preferences or if innovation has a negative bias on the survival rate of firms, Fisher's runaway process that is stably converging towards the equilibrium can be 
recovered.

\section{Conclusion}

This paper has discussed the interaction between supply and demand in the development of innovation, based on the framework of evolutionary ecology and population genetics. In particular, we have focused on Fisher's runaway process representing the fact that more than two characteristics coevolve. All propositions that have been established can be summarized in Table 1 .

$<$ Table 1>

Only with the survival rate of firms, Fisher's process becomes one of convergence towards the stable equilibrium. However, if the level of satisfaction of consumers is added to the model, then Fisher's process is regarded as a temporally disequilibrium process at best. These conclusions can aptly explain why many new technologies/services could not overcome the start-up problems. However, with another condition, such as the existence of power-users or the negative bias of innovation, Fisher's process can be recovered as evolutionarily stable.

In conclusion, we have obtained interesting results concerning Fisher's runaway process of innovation: there are various types of process in which the quality 
of products and the preferences of consumers coevolve; without specific conditions, stably converging coevolution will not be fulfilled.

\section{Appendix I. The Derivation of the fundamental equation}

The fundamental equation (4) can be derived in the following way. First of all, we define the basic variables as follows:

the number of the products of quality $x$ that a consumer with preference $y$ discovers in a period:

$$
n_{x y}
$$

the total number of the products that consumers discover in a period: $N=\sum \sum_{x, y} n_{x y}$ the average value of the quality of products: $E_{x}=\sum \sum_{x, y} x n_{x y} / N$

the variance of the quality of products: $V_{x}=\sum \sum_{x, y}\left(x-E_{x}\right)^{2} n_{x y} / N$

the average value of the quality of the products that can survive in the next period:

$$
E_{x,+1}=\sum \sum_{x, y} x S_{x} n_{x y} / \sum \sum_{x, y} S_{x} n_{x y}
$$

At this point, defining the survival rate of a firm as $S_{x}=S(x, y)$, the change of the average value of the quality of product, $E_{x}$, can be expressed as follows:

$$
\begin{aligned}
\Delta E_{x}= & E_{x,+1}-E_{x} \\
= & \sum \sum_{x, y} x S(x, y) n_{x y} / \sum \sum_{x, y} S(x, y) n_{x y}-\sum \sum_{x, y} x n_{x y} / N \\
= & {\left[\sum \sum_{x, y} x S(x, y) n_{x y} / N-\left(\sum \sum_{x, y} x n_{x y} / N\right)\left(\sum \sum_{x, y} S(x, y) n_{x y} / N\right)\right] } \\
& /\left(\sum \sum_{x, y} S(x, y) n_{x y} / N\right) \\
= & \operatorname{Cov}(x, S(x, y)) / E(S(x, y)) .
\end{aligned}
$$

Note here that $\operatorname{Cov}$ denotes variance and $E$ denotes expectation. Next, by applying a Taylor expansion to $S(x, y)$ around $\left(E_{x}, E_{y}\right)$, the following equation is obtained: 


$$
\begin{aligned}
& \operatorname{Cov}(x, S(x, y))=\operatorname{Cov}\left(x, S\left(E_{x}, E_{y}\right)+\partial S(x, y) / \partial x\left(x-E_{x}\right)+\partial S(x, y) / \partial y\left(y-E_{y}\right)\right) \\
& =S\left(E_{x}, E_{y}\right) \operatorname{Cov}(x, 1)+(\partial S(x, y) / \partial x) \operatorname{Cov}\left(x, x-E_{x}\right)+(\partial S(x, y) / \partial y) \operatorname{Cov}\left(x, y-E_{y}\right) \\
& =V_{x}(\partial S(x, y) / \partial x)+R(\partial S(x, y) / \partial y) \\
& \left(\because \operatorname{Cov}(x, 1)=0, \operatorname{Cov}\left(x, x-E_{x}\right)=V_{x}, \operatorname{Cov}\left(x, y-E_{y}\right)=R\right) \text {. }
\end{aligned}
$$

Furthermore, by applying a Taylor expansion to $E(S(x, y))$ around $\left(E_{x}, E_{y}\right)$, the following equation is obtained:

$$
\begin{aligned}
& E(S(x, y))=E\left(S\left(E_{x}, E_{y}\right)+(\partial S(x, y) / \partial x)\left(x-E_{x}\right)+(\partial S(x, y) / \partial y)\left(y-E_{y}\right)\right. \\
& \quad=S\left(E_{x}, E_{y}\right)+(\partial S(x, y) / \partial x) E\left(x-E_{x}\right)+(\partial S(x, y) / \partial y) E\left(y-E_{y}\right) \\
& \quad=S\left(E_{x}, E_{y}\right) \\
& \left(\because E\left(x-E_{x}\right)=E\left(y-E_{y}\right)=0\right) .
\end{aligned}
$$

From what has been stated, the following equation is obtained concerning the average quality of products, $E_{x}$ :

$$
\begin{aligned}
\Delta E_{x} & =\operatorname{Cov}(x, S(x, y)) / E(S(x, y)) \\
& =\left[V_{x}(\partial S(x, y) / \partial x)+R(\partial S(x, y) / \partial y)\right] / S\left(E_{x}, E_{y}\right) \\
& =V_{x}(\partial \log S(x, y) / \partial x)+\left.R(\partial \log S(x, y) / \partial y)\right|_{x=E x, y-E y} .
\end{aligned}
$$

Similarly, the following equation is obtained concerning the average preference of consumers, $E_{y}$ :

$$
\Delta E_{y}=R(\partial \log D(x, y) / \partial x)+\left.V_{y}(\partial \log D(x, y) / \partial y)\right|_{x=E x, y=E y} .
$$

\section{Appendix II. The analysis of the equilibrium}

The eigenvalue of matrix $\left(\begin{array}{cc}0 & a V_{x} \\ 0 & a R\end{array}\right)$ corresponding to Eqs. (5) is $\{0, a R\}$; the equilibrium of Eqs. (5) is unstable because of $a R>0$. The eigenvalue of matrix $\left(\begin{array}{cc}-2 b V_{x} & a V_{x} \\ -2 b R & a R\end{array}\right)$ corresponding to Eqs. (8) is $\{0, a R-2 b V x\}$; the equilibrium of Eqs. (8) is stable if $a R-2 b V x<0$, while unstable if $a R$ $2 b V x>0$. The eigenvalue of matrix $\left(\begin{array}{cc}-2 b V_{x} & a V_{x}-2 c R \\ -2 b R & a R-2 c V_{y}\end{array}\right)$ corresponding to Eqs. (10) is 
$\frac{1}{2}\left(a R-2 b V_{x}-2 c V_{y} \pm \sqrt{\left(a R-2 b V_{x}-2 c V_{y}\right)^{2}+16 b c\left(V_{x} V_{y}-R^{2}\right)}\right) ;$ the sign of the eigenvalue depends on parameters; eigenvalues are $\{-0.169636,-0.00536444\}$ in the case of $a=b=0.5, c=0.0125$, $d=0.05, V_{x}=V_{y}=0.2, R=0.06$, as assumed in Fig. 7, and therefore the equilibrium of Eqs. (10) is stable. The same analysis can be applied to Eqs. (12) and (13).

\section{References}

Andersen B (2001) Technological Change and the Evolution of Corporate Innovation. Edward Elgar

Anderson ES (1994) Evolutionary Economics: Post Schmperiterian Contributions. Pinter.

Antonelli G, De Liso N (eds.) (1997) Economics of structural and Technological Change. Routledge

Bulmer MG (1985) The Mathematical Theory of Quantitative Genetics. Oxford University Press

Dosi G, Freeman C, Nelson R, Silverberg G, Soete L (1988) Technical Change and Economic Theory. Pinter

Dosi G (2000) Innovation, Organization and Economic Dynamics. Edward Elgar.

Fisher RA (1915) The Evolution of Sexual Preferences. Eugenics Review 7: 184-192

Flconer DS (1981) Introduction to Quantitative Genetics. Longman

Freeman C (1982) The Economics of Industrial Innovation. Frances Printer 
Grupp H (1998) Foundations of the Economics of Innovation: Theory, Measurement and Practice. Edward Elgar

Hodgson GM (1994) Economics and Evolution. Polity Press

Iwasa Y, Pomiankowski A, Nee S (1991) The Evolution of Costly Mate Preference II. Evolution 45: $1431-1442$

Kirkpatrick M (1985) Evolution of Female Choice and Male Parental Investment in Polygynous Species: the Demise of the Sexy Son. The American Naturalist 125: $788-810$

Kirkpatrick M (1986) The Handicap Mechanism of Sexual Selection Does Not Work. The American Naturalist 127: 222-240

Lande R (1981) Models of Speciation by Sexual Selection on Polygenic Traits. Proceedings of Natural Academic Science of the U.S.A. 78: 3721-3725

Mayr E (1959) Typological versus Population Thinking. Reprinted in Mayr E (1976)

Evolution and the Diversity of Life: Selected Essays. Belknap Press

Metcalfe JS (1998) Evolutionary Economics and Creative Destruction. Routledge

Nelson R,Winter S (1984) An Evolutionary Theory of Economic Change. Harvard University Press

Pomiankowski A, Iwasa Y, Nee S (1991) The Evolution of Costly Mate Preference I. Evolution 45: 1422-1430

Rohlfs JH (2001) Bandwagon Effects in High-Technology Industries. The MTT Press Sundbo J (1998) The Theory of Innovation: Entrepreneurs. Technology and Strategy. Edward Elgar 
Witt U (1993) Evolutionary Economics. Edward Elgar 
Fig. 1. The timing of the model in one period

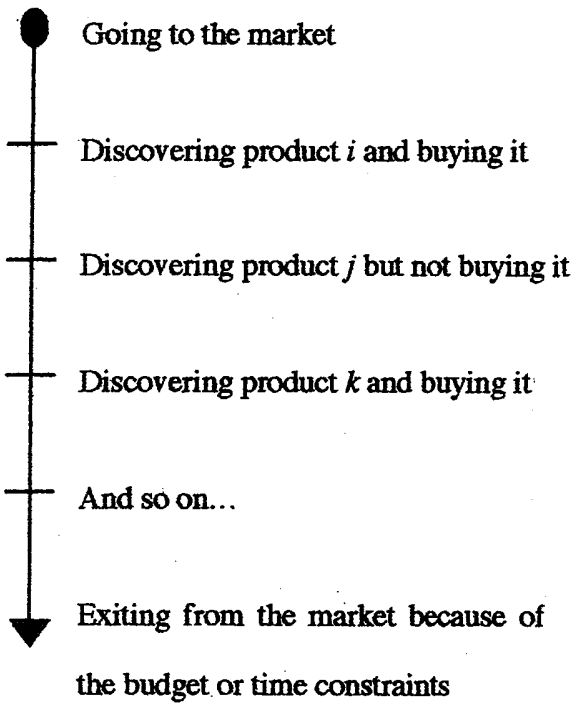

Fig. 2. Fisher's runaway process

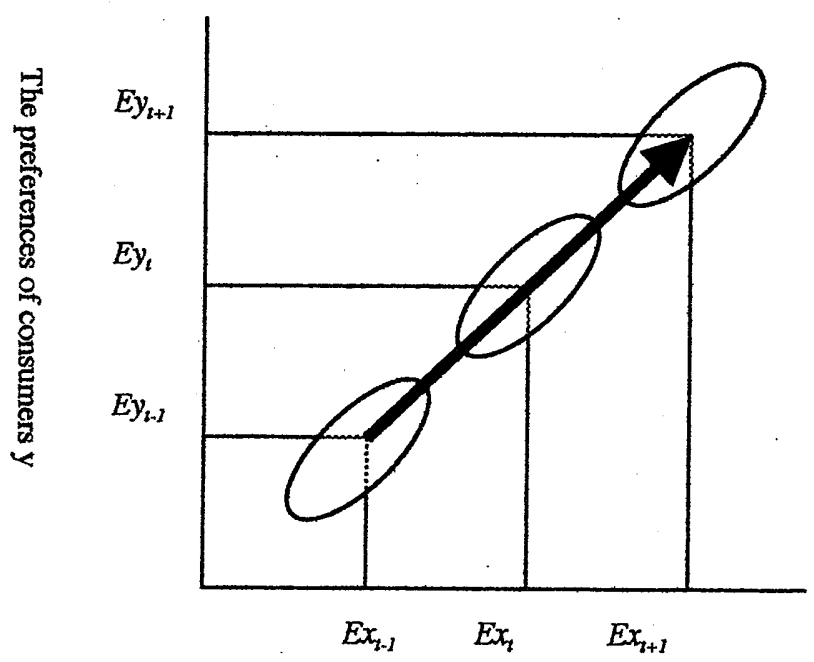

The quality of products $x$ 
Fig. 3. Evolutionarily unstable runaway

given $a=0.5, V_{x}=0.2, R=0.06$

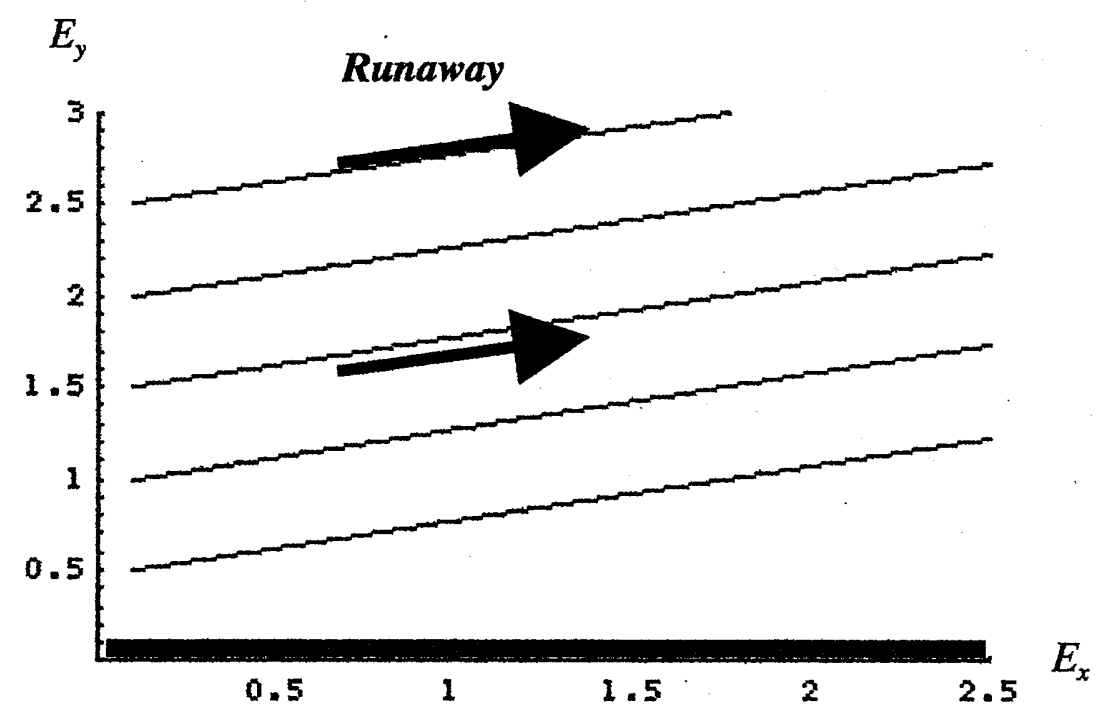

Fig. 4. Evolutionarily stable runaway

given $a=b=0.5, V_{x}=0.2, R=0.06$

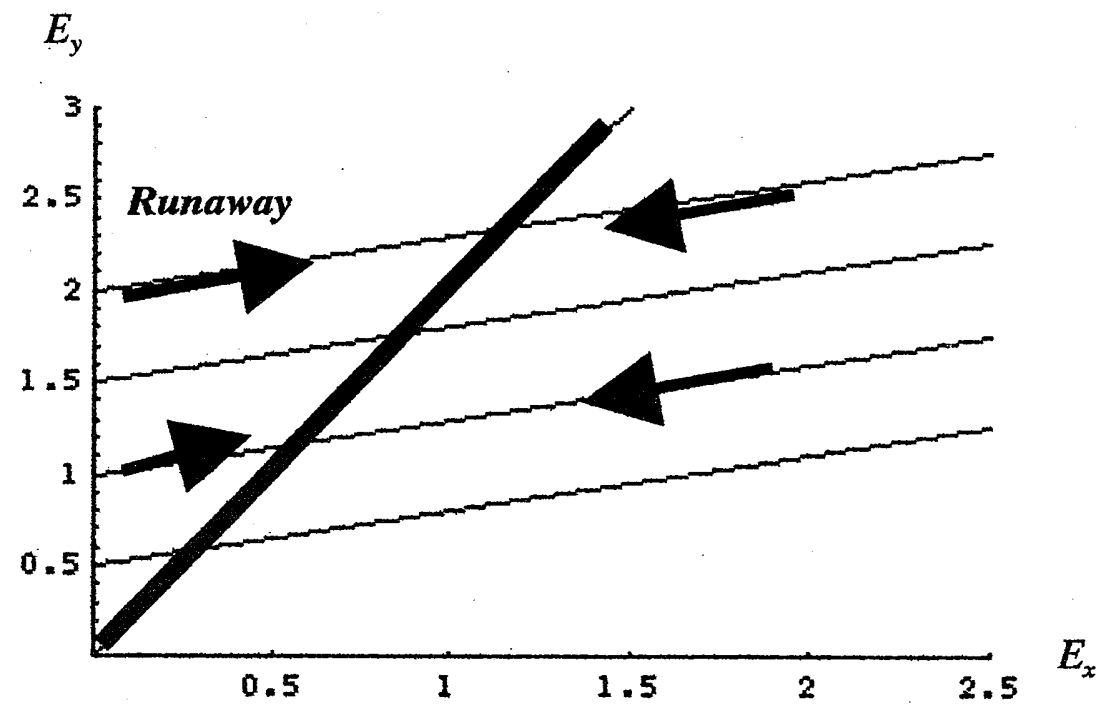


Fig. 5. Temporally dis-equilibrium runaway

given $a=b=0.5, c=0.0125, V_{x}=V_{y}=0.2, R=0.06$

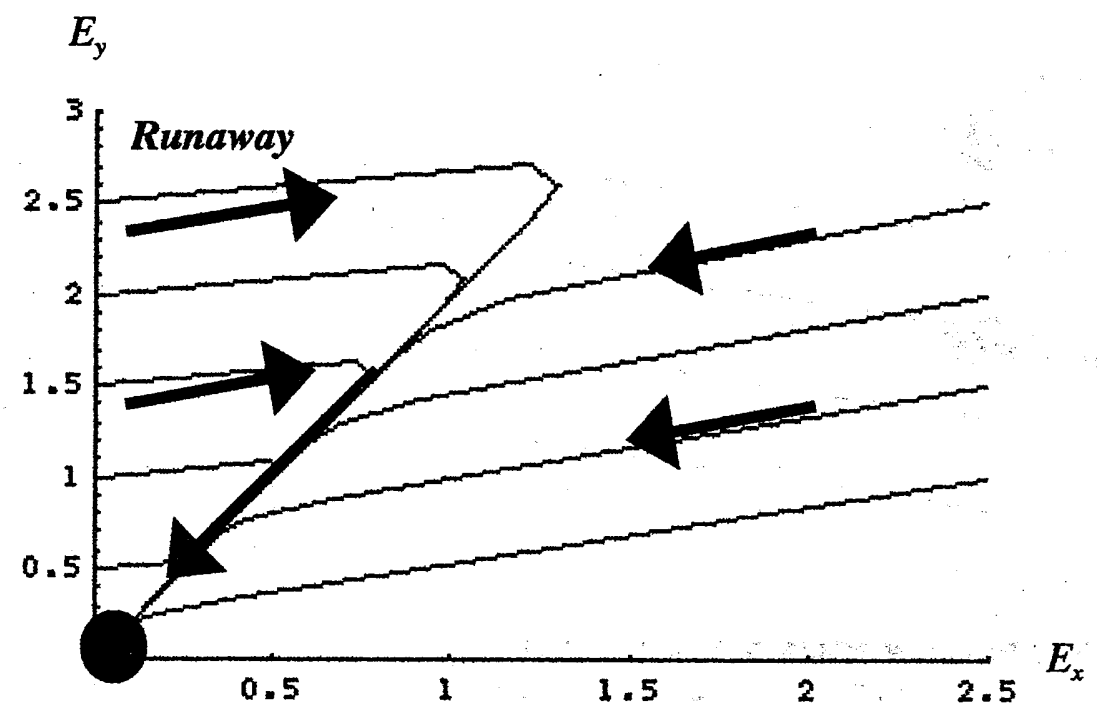

Fig. 6. An example of a trajectory depicted in Fig. 5

given $E_{x}(t=0)=0, E_{y}(t=0)=2$

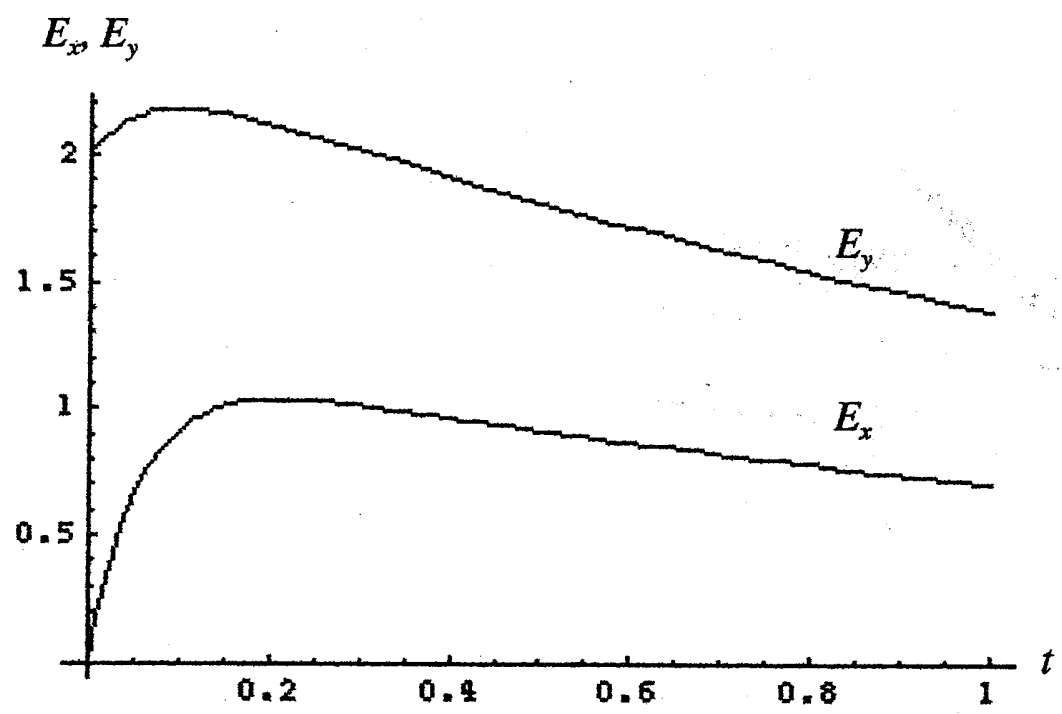


Fig. 7. Evolutionarily stable runaway

given $a=b=0.5, c=0.0125, d=0.05, V_{x}=V_{y}=0.2, R=0.06$

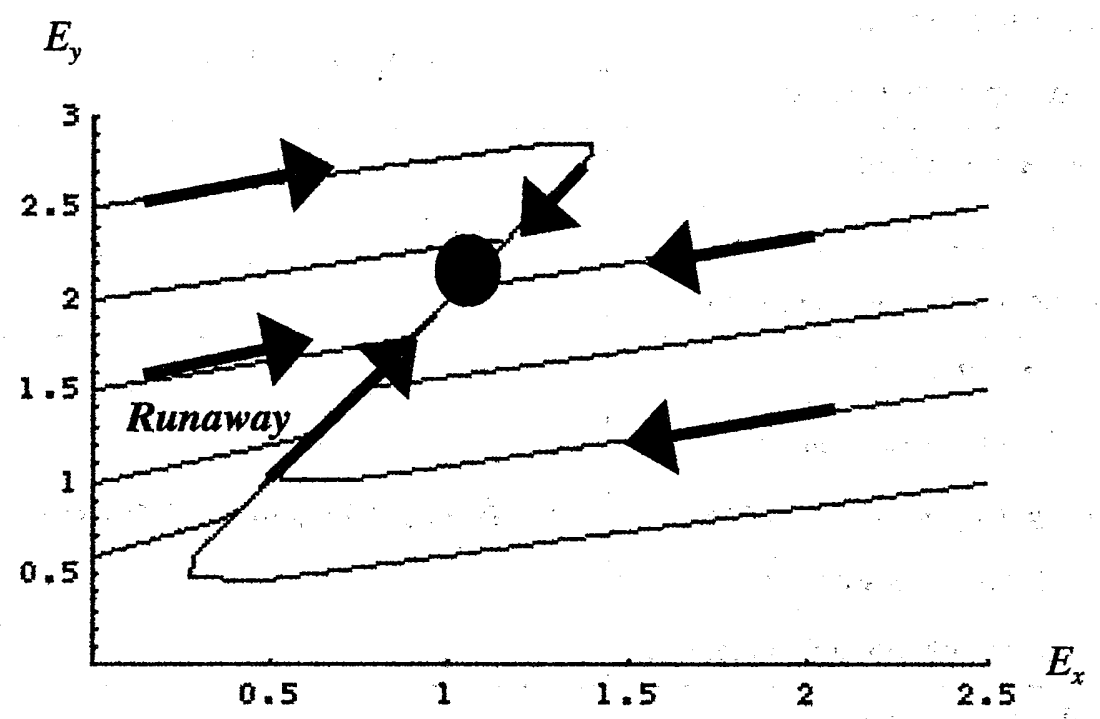

Fig. 8. An example of a trajectory depicted in Fig. 7 given $E_{x}(t=0)=0, E_{y}(t=0)=1$

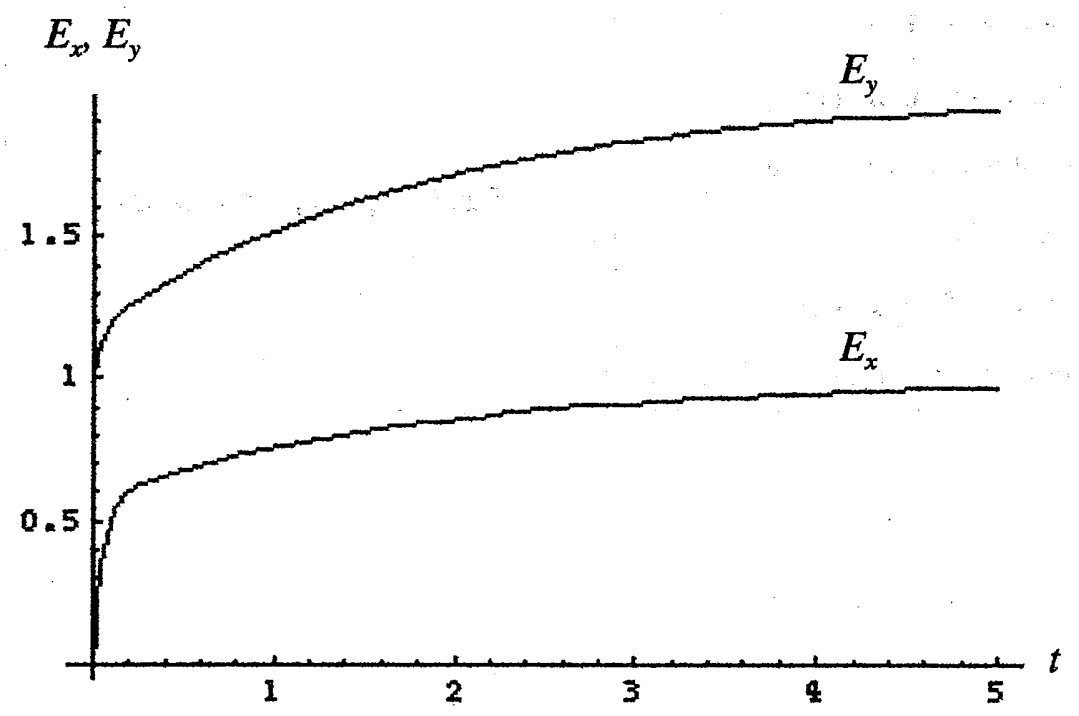


Table 1. Various aspects of Fisher's runaway process

\begin{tabular}{|c|c|}
\hline Factors of the model & Fisher's runaway process \\
\hline The survival rate of a firm: & Unstably diverging process \\
\hline (1) the interaction of supply and demand & Stably converging process \\
\hline (1) the interaction of supply and demand; & \\
(2) the cost for improving the quality of products & The survival rate of a firm: \\
\hline (1) the interaction of supply and demand; & Temporally dis-equilibrium process \\
(2) the cost for improving the quality of products & \\
The level of satisfaction of consumers: & \\
(1) the misfortune of a consumer with too-high preference & \\
\hline The survival rate of a firm: & Stably converging process \\
(1) the interaction of supply and demand; & \\
(2) the cost for improving the quality of products & \\
The level of satisfaction of consumers: & \\
The level of satisfaction of consumers: & \\
(1) the misfortune of a consumer with too-high preference; & \\
(2) the existence of power-users & \\
(1) the interaction of supply and demand; & \\
(2) the cost for improving the quality of products; & \\
\hline
\end{tabular}

